\title{
Prediction of early vascular cement leakage following percutaneous vertebroplasty in spine metastases: the Peking University First Hospital Score (PUFHS)
}

Xuedong Shi ${ }^{1^{*}+}$, Yunpeng Cui ${ }^{1+}$, Yuanxing Pan ${ }^{1}$, Bing Wang ${ }^{1}$ and Mingxing Lei ${ }^{2,3^{*}}$

\begin{abstract}
Background: Cement leakage into venous blood posed significant challenge to surgeons. The aim of the study was to create a Peking University First Hospital Score (PUFHS) which could evaluate the probability of vascular cement leakage among spine metastases patients following percutaneous vertebroplasty.

Methods: The study retrospectively enrolled 272 spine metastases patients treated with percutaneous vertebroplasty. We randomly extracted all enrolled patients as the training or validation group and baseline characteristic comparison was assessed between the two groups. Creation of the PUFHS was performed in the training group and validation of the PUFHS was performed in the validation group.

Results: Of all the 272 patients, the total number of included vertebrae was 632 and the median treated levels were 2 per patient. Vascular cement leakage occurred in 26.47\% (72/272) of patients. The baseline characteristics were comparable between the two groups $(P>0.05)$. Three risk predictors (primary cancer types, number of treated vertebrae levels, and vertebrae collapse) were included in the PUFHS. The area under the receiver operating characteristic curve (AUROC) of the PUFHS was 0.71 in the training group and 0.69 in the validation group. The corresponding correct classification rates were 73.0 and $70.1 \%$, respectively. The calibration slope was 0.78 (95\% confidence interval[Cl]: $0.45-1.10)$ in the training group and 1.10 (95\% Cl: $0.73-1.46)$ in the validation group. The corresponding intercepts were 0.06 (95\% Cl: $-0.04-0.17)$ and -0.0079 (95\% Cl: $-0.11-0.092)$, respectively.
\end{abstract}

Conclusions: Vascular cement leakage is common among spine metastases after percutaneous vertebroplasty. The PUFHS can calculate the probability of vascular cement leakage, which can be a useful tool to inform surgeons about vascular cement leakage risk in advance.

Keywords: Spine metastases, Percutaneous vertebroplasty, Vascular cement leakage, Prediction score

\footnotetext{
*Correspondence: xuedongs@hotmail.com; 825979020@qq.com

${ }^{+}$Xuedong Shi and Yunpeng Cui contributed equally to this work.

'Department of Orthopedic Surgery, Peking University First Hospital, No.8

Xishiku Street, Xicheng District, Beijing 100032, China

2Department of Orthopedic Surgery, Hainan Hospital of Chinese PLA General

Hospital, Haitang District, Jianglin Rd, Sanya 572013, China

Full list of author information is available at the end of the article
}

(c) The Author(s). 2021 Open Access This article is licensed under a Creative Commons Attribution 4.0 International License, which permits use, sharing, adaptation, distribution and reproduction in any medium or format, as long as you give appropriate credit to the original author(s) and the source, provide a link to the Creative Commons licence, and indicate if changes were made. The images or other third party material in this article are included in the article's Creative Commons licence, unless indicated otherwise in a credit line to the material. If material is not included in the article's Creative Commons licence and your intended use is not permitted by statutory regulation or exceeds the permitted use, you will need to obtain permission directly from the copyright holder. To view a copy of this licence, visit http://creativecommons.org/licenses/by/4.0/ The Creative Commons Public Domain Dedication waiver (http://creativecommons.org/publicdomain/zero/1.0/) applies to the data made available in this article, unless otherwise stated in a credit line to the data. 


\section{Background}

Spine metastases occur in $5-10 \%$ of all malignant tumors during disease course [1], and the incidence of spine metastases is also increasing as systemic therapies for cancer patients improve. Spine metastases are often characterized by severe back pain and vertebral compression fractures, which may lead to declining mobility, kyphosis, and even neurologic compression [2]. If those patients are left untreated, they may suffer from poor quality of life.

The therapeutic strategies for spine metastases are usually conventional palliative, aiming at improving patient's quality of remaining life and emphasizing in reducing pain and improving or, perhaps at most cases, just maintaining function status. Although open surgery is capable of realizing fully decompression and/or total removal of spine metastatic lesions, it can also bring out large trauma, severe complications, and delays in systematic treatment of the primary tumor. Notably, it is not appropriate for multiple spine metastases to receive open surgery [3]. Therefore, percutaneous vertebroplasty, a minimally invasive procedure, was developed to treat spine metastases, which has become one of the fastest emerging procedures in spine surgery [4]. This technique consists of percutaneous injection of polymethylmethacrylate (PMMA) into vertebrae body through transpedicular approach, which is proved to be an effective technique for promptly obtaining pain relief, controlling local tumor burden, preventing further vertebral collapse or spinal cord compression, and facilitating the return to early systemic and radiation therapy $[5,6]$.

However, despite the fact that percutaneous vertebroplasty is minimal invasive, complications can still occur during surgery. PMMA leakage is considered as the major cause of complications. Cement leakage into venous blood vessels and/or the spinal canal may result in serious consequences, though the majority of cement leakage would not cause any symptoms [7]. Pulmonary embolism can be caused by PMMA leaking into blood vessels and the incidence varies from 4.6 to $23.0 \%$ [8]. A systematic review revealed serious complication rates ranged from 2 to $11.5 \%$ [9]. Intracardiac cement embolism could be up to $3.9 \%$ [10]. Literature reported leaked cement pulmonary embolism and cardiac perforation was regarded as a cause of unexpected death following percutaneous vertebroplasty [11]. Thus, appropriate strategies to guide PMMA injections are really warranted to prevent vascular cement leakage and subsequent life-threatening complications.

Therefore, the aim of the study was to identify potential risk factors for predicting vascular cement leakage in spine metastases following percutaneous vertebroplasty and further create the Peking University First Hospital Score (PUFHS) to evaluate the probability of vascular cement leakage so as to realize early detection of this complication.

\section{Methods \\ Patients}

We retrospectively enrolled 272 spine metastases patients treated with percutaneous vertebroplasty at the orthopedic department of the Peking University First Hospital between January 2010 and January 2019. Patients were included if they (1) had an age of more than 50 years old, (2) had mixed or osteolytic metastatic vertebrae lesions, (3) had serious or uncontrolled back pain, and (4) received percutaneous vertebroplasty; Patients were excluded if they (1) had intramedullary metastases, (2) vertebrae compression fracture due to primary spine tumor, trauma, osteoporosis, and/or angioma, (3) received combined therapy (i.e thermal ablation + vertebroplasty), and (4) skin infections at patient's corresponding involved vertebrae. If a patient's serious uncontrolled back pain was not significantly relieved after conservative treatments, they were also considered to be performed with percutaneous vertebroplasty; If patients had severe radiculopathy and deteriorated function and were tolerable to open surgery, decompressive surgery and spine stabilization was recommended to these patients; If patients performed with more than one percutaneous vertebroplasty at different time, the second and subsequent operations were not analyzed in the study. This study was performed based on the Declaration of Helsinki. The Ethics Committee Board of the Peking University First Hospital approved the study and waived patient's consent form due to anonymized and retrospective data.

\section{Study design}

We randomly extracted $3 / 4$ of all included patients as the training group $(n=204)$ or validation group $(n=$ 204), respectively. Baseline characteristic comparison was evaluated between the training and validation group. Creation of the PUFHS was performed in the training group and the validation of the PUFHS was performed in the validation group.

\section{Primary outcome}

Vascular cement leakage was defined that cement leaks into veins, including anterior external venous plexus or basivertebral veins, which was evaluated using intraoperative fluoroscopy images (mobile $\mathrm{C}$-arm) or postoperative CT examination and X-ray. Cement pulmonary embolism was a particular type of the vascular cement leakage which was assessed based on chest radiographs and/or CT examinations, if patient's pulmonary symptoms were presented after surgery. If patients had radicular pain, neurological deficits, and/or dyspnea 
because of vascular cement leakage, researchers would record the corresponding clinical symptoms. Figure 1 shows a case report. The bone cement mainly used in the study was Mendec Spine Resin and Kit (Tecres S.P.A. Italy).

\section{Potential risk predicators}

Eleven potential risk predictors were collected and analyzed for evaluating vascular cement leakage in spine metastases following percutaneous vertebroplasty. The potential risk factors included basic information, such as age (mean, years), gender (male vs. female), primary cancer types (rapid vs. moderate vs. slow) [12], therapeutic information, including preoperative treatments (topical treatments vs. systematic treatments vs. no treatment), and radiographic data, such as the number of treated vertebrae levels ( 1 vs. 2 vs. 3 vs. $\geq 4$ ), vertebrae collapse (no collapse vs. less than $50 \%$ vs. more than 50\%) [13], cortical osteolytic destruction in posterior wall (yes vs. no), vertebral endplate fracture (yes vs. no), the Bilsky scale (0 vs. 1 vs. 2 vs. 3) [14], appearance of spine metastases (mixed lesions vs. osteolytic lesions), and loadbearing lines of spine (normal vs. abnormal). Patient's age was defined as the time interval between patient's birth data and surgery data. Rapid growth cancers were defined as patients diagnosed with lung cancer, esophageal cancer, stomach cancer, liver cancer, pancreatic cancer, colon cancer, and unknown cancer, moderate growth cancers were kidney cancer and uterus caner, and slow growth cancers were breast cancer, thyroid cancer, prostate cancer, and others. The Bilsky scale was used to evaluate the severity of spinal cord compression: a higher score represented a severer spinal cord compression. Preoperative treatments were classified into topical treatments (topical analgesics and radiotherapy), systematic treatments (oral or intravenous analgesics, targeted drugs, and chemotherapy), and no treatment.

\section{Creating the PUFHS}

In the training group, the Least Absolute Shrinkage and Selection Operator (LASSO) method was used to identify the above-mentioned potential risk predictors, and significant predictors were included in the PUFHS. The coefficients of the included risk predictors were assigned based on the estimates obtained from the multiple logistic regression analysis. The PUFHS would be created as follows: $\mathrm{P}(\mathrm{Y}=1)=e^{\text {intercept }+a x 1+b x 2+c \times 3} /\left(1+e^{\text {intercept }+}\right.$ $\left.{ }^{a x} 1+b x 2+c \times 3\right)$. In the PUFHS, $\mathrm{a}, \mathrm{b}$, and $\mathrm{c}$ were coefficients of the included risk predictors. Intercept was also calculated according to the multiple logistic regression analysis. $\mathrm{P}(\mathrm{Y}=1)$ represented the predicted probability of vascular cement leakage.

\section{Validating the PUFHS}

Validation of the PUFHS was performed in the validation and training group. The discrimination and calibration ability was used to evaluate the predictive performance of the PUFHS. The discrimination ability was defined as the capability that the PUFHS could distinguish patients with vascular cement leakage from patients without this complication. The calibration ability was defined as the consistence between the PUFHSpredicted probability of vascular cement leakage and the actual observed probability of vascular cement leakage.

The PUFHS's discrimination ability was evaluated using the area under the receiver operating characteristic (AUROC) curve and discrimination slope. A C-value of more than 0.6 from the AUROC curve indicated useful model and 0.7 indicated good model. Discrimination slope was the difference between the PUFH-predicted
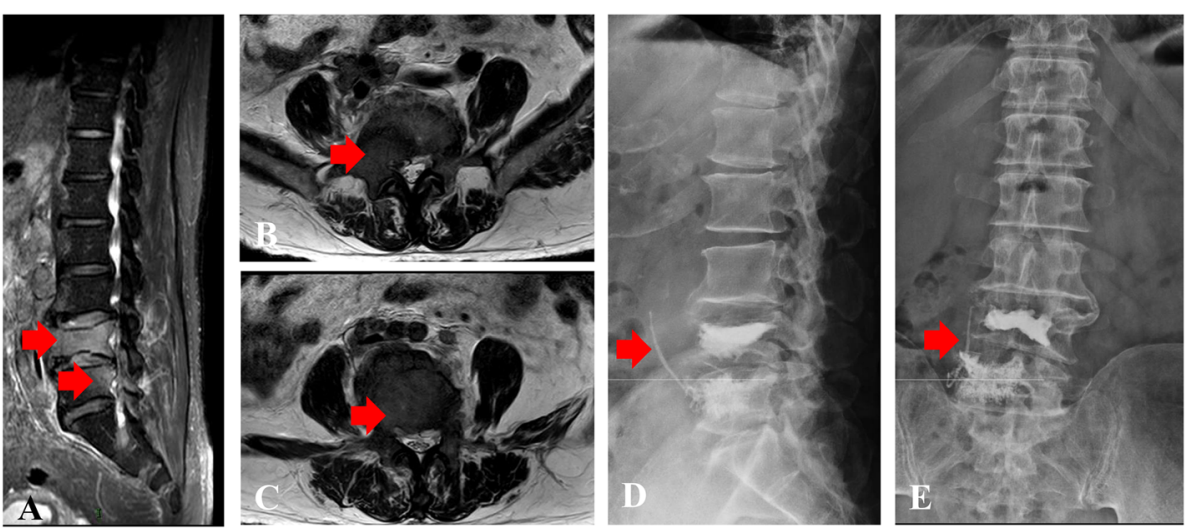

Fig. 1 An 80-year-old female with spine metastases treated with percutaneous vertebroplasty. A Preoperative sagittal lumbar vertebra MRI (T2) showed spine metastases at L4 and L5; B Preoperative transversal MRI (T2) showed metastatic lesion at L4; C. Preoperative transversal MRI (T2) showed metastatic lesion at L5; The red arrow indicates metastatic tumor in (A, B, and C). D Postoperative lateral lumbar vertebra X-ray presented bone cement at $L 4$ and $L 5$ and vascular cement leakage; $\mathbf{E}$ Postoperative anteroposterior lumbar vertebra X-ray presented bone cement at $L 4$ and L5 and vascular cement leakage. The red arrow indicates vascular cement leakage in (D and $\mathbf{E})$ 
Table 1 Patient's basic characteristics

\begin{tabular}{|c|c|}
\hline Characteristics & Patients $(n=272)$ \\
\hline Age (years, mean $\pm S D$ ) & $67.92 \pm 10.21$ \\
\hline \multicolumn{2}{|l|}{ Gender } \\
\hline Male & $59.93 \%(163 / 272)$ \\
\hline Female & $40.07 \%(109 / 272)$ \\
\hline \multicolumn{2}{|l|}{ Primary cancer types } \\
\hline Slow growth & $28.31 \%(77 / 272)$ \\
\hline Moderate growth & $15.44 \%(42 / 272)$ \\
\hline Rapid growth & $56.25 \%(153 / 272)$ \\
\hline \multicolumn{2}{|l|}{ Preoperative treatments } \\
\hline Topical treatments & $36.40 \%(99 / 272)$ \\
\hline Systematic treatments & $32.72 \%(89 / 272)$ \\
\hline No treatment & $30.88 \%(84 / 272)$ \\
\hline \multicolumn{2}{|l|}{ Number of treated vertebrae levels } \\
\hline 1 & $37.87 \%(103 / 272)$ \\
\hline 2 & $26.10 \%(71 / 272)$ \\
\hline 3 & $16.18 \%(44 / 272)$ \\
\hline$\geq 4$ & $19.85 \%(54 / 272)$ \\
\hline \multicolumn{2}{|l|}{ Vertebrae collapse } \\
\hline No collapse & $61.76 \%(168 / 272)$ \\
\hline Less than $50 \%$ & $24.63 \%(67 / 272)$ \\
\hline More than $50 \%$ & $13.60 \%(37 / 272)$ \\
\hline \multicolumn{2}{|c|}{ Cortical osteolytic destruction in posterior wall } \\
\hline Yes & $41.91 \%(114 / 272)$ \\
\hline No & $58.09 \%(158 / 272)$ \\
\hline \multicolumn{2}{|l|}{ Vertebral endplate fracture } \\
\hline Yes & $17.65 \%(48 / 272)$ \\
\hline No & $82.35 \%(224 / 272)$ \\
\hline \multicolumn{2}{|l|}{ The Bilsky scale } \\
\hline 0 & $77.21 \%(210 / 272)$ \\
\hline 1 & $10.29 \%(28 / 272)$ \\
\hline 2 & $10.66 \%(29 / 272)$ \\
\hline 3 & $1.84 \%(5 / 272)$ \\
\hline \multicolumn{2}{|l|}{ Appearance of spine metastases } \\
\hline Mixed lesions & $17.65 \%(48 / 272)$ \\
\hline Osteolytic lesions & $82.35 \%(224 / 272)$ \\
\hline \multicolumn{2}{|l|}{ Load-bearing lines of spine } \\
\hline Normal & $88.24 \%(240 / 272)$ \\
\hline Abnormal & $11.76 \%(32 / 272)$ \\
\hline \multicolumn{2}{|l|}{ Vascular cement leakage } \\
\hline Yes & $26.47 \%(72 / 272)$ \\
\hline No & $73.53 \%(200 / 272)$ \\
\hline The total number of included vertebrae & 632 \\
\hline \multicolumn{2}{|l|}{ Sites of vertebra } \\
\hline Cervical & $1.27 \%(8 / 632)$ \\
\hline
\end{tabular}

Table 1 Patient's basic characteristics (Continued)

\begin{tabular}{ll}
\hline Characteristics & Patients $(\boldsymbol{n}=\mathbf{2 7 2})$ \\
\hline Thoracic & $46.04 \%(291 / 632)$ \\
Lumbar & $46.04 \%(291 / 632)$ \\
Sacral & $6.65 \%(42 / 632)$ \\
\hline Abbreviations: SD standard deviation &
\end{tabular}

mean probability with vascular cement leakage (positive events) and without it (negative events).

The calibration ability of the PUFHS was evaluated using the calibration slope and goodness-of-fit test. Calibration slope was measure by plotting deciles of the predicted probability of vascular cement leakage against the observed proportions in each decile. A smooth line $(\mathrm{Y}=$ $\mathrm{ax}+\mathrm{b}$ ) was fitted using linear regression analysis in the Microsoft Excel software. In the smooth line, 'a' indicated the calibration slope and ' $b$ ' indicated the intercept. In ideal circumstance, the closer calibration slope is to 1 , the better it is; the closer intercept is close to 0 , the better it is. A $P$-value of more than 0.05 obtained from the goodness-of-fit text indicated good calibration.

\section{Statistical analysis}

Analyses were performed in SAS 9.2 software and R version 3.5.3 for Windows XP. Continuous variables were presented as mean \pm SD. The characteristic differences between the training and validation group were analyzed based on the chi-square test and $t$ or rank test. The difference between the three risk groups was compared using the Kruskal Wallis test and the Chi-square test. The calibration slope and intercept was calculated using the Microsoft Excel software. A $P$-value of less than 0.05 was considered as statistical significance.

\section{Results}

\section{Patient's demographics}

Of all the 272 patients, the mean age was $67.92 \pm 10.21$ years (Table 1). The majority of patients were male $(59.93 \%, 163 / 272)$ and diagnosed with rapid growth cancers $(56.25 \%, 153 / 272)$, followed by slow growth cancers $(28.31 \%, 77 / 272)$. In detail, $33.82 \%(92 / 272)$ of patients had lung cancer, $16.18 \%$ (44/272) had prostate cancer, and $11.40 \%(31 / 272)$ had renal cancer. The number of patients received topical treatments $(36.40 \%, 99 / 272)$ was similar with patients treated with systematic treatments $(32.72 \%, 89 / 272)$ or no treatment $(30.88 \%, 84 /$ 272).

Regarding radiographic data, the total number of included vertebrae was 632 and among them 1.27\% (8/ 632) was cervical vertebra, $46.04 \%(291 / 632)$ was thoracic vertebra, $46.04 \%(291 / 632)$ was lumbar vertebra, and $6.65 \%(42 / 632)$ was sacral vertebrae. The majority of patients had only one treated vertebrae (37.87\%, 103/ 
Table 2 The characteristic comparison of patients in the training and validation group

\begin{tabular}{|c|c|c|c|}
\hline Characteristics & The training group $(n=204)$ & The validation group $(n=204)$ & $P$-values \\
\hline Age (years, mean $\pm S D$ ) & $67.78 \pm 10.12$ & $67.85 \pm 10.44$ & 0.95 \\
\hline \multicolumn{4}{|l|}{ Gender } \\
\hline Male & 121 & 129 & \multirow[t]{2}{*}{0.42} \\
\hline Female & 83 & 75 & \\
\hline \multicolumn{4}{|l|}{ Primary cancer types } \\
\hline Slow growth & 56 & 60 & \multirow[t]{3}{*}{0.90} \\
\hline Moderate growth & 31 & 31 & \\
\hline Rapid growth & 117 & 113 & \\
\hline \multicolumn{4}{|l|}{ Preoperative treatments } \\
\hline Topical treatments & 74 & 72 & \multirow[t]{3}{*}{0.98} \\
\hline Systematic treatments & 70 & 71 & \\
\hline No treatment & 60 & 61 & \\
\hline \multicolumn{4}{|l|}{ Number of treated vertebrae levels } \\
\hline 1 & 76 & 80 & \multirow[t]{4}{*}{0.77} \\
\hline 2 & 47 & 53 & \\
\hline 3 & 36 & 31 & \\
\hline$\geq 4$ & 45 & 40 & \\
\hline \multicolumn{4}{|l|}{ Vertebrae collapse } \\
\hline No collapse & 121 & 124 & \multirow[t]{3}{*}{0.77} \\
\hline Less than $50 \%$ & 56 & 50 & \\
\hline More than $50 \%$ & 27 & 30 & \\
\hline \multicolumn{4}{|c|}{ Cortical osteolytic destruction in posterior wall } \\
\hline Yes & 86 & 88 & \multirow[t]{2}{*}{0.84} \\
\hline No & 118 & 116 & \\
\hline \multicolumn{4}{|l|}{ Vertebral endplate fracture } \\
\hline Yes & 39 & 37 & \multirow[t]{2}{*}{0.80} \\
\hline No & 165 & 167 & \\
\hline \multicolumn{4}{|l|}{ The Bilsky scale } \\
\hline 0 & 157 & 156 & \multirow[t]{4}{*}{0.96} \\
\hline 1 & 22 & 20 & \\
\hline 2 & 20 & 23 & \\
\hline 3 & 5 & 5 & \\
\hline \multicolumn{4}{|l|}{ Appearance of spine metastases } \\
\hline Mixed lesions & 33 & 37 & \multirow[t]{2}{*}{0.60} \\
\hline Osteolytic lesions & 171 & 167 & \\
\hline \multicolumn{4}{|l|}{ Load-bearing lines of spine } \\
\hline Normal & 180 & 176 & \multirow[t]{2}{*}{0.55} \\
\hline Abnormal & 24 & 28 & \\
\hline \multicolumn{4}{|l|}{ Vascular cement leakage } \\
\hline Yes & 57 & 51 & \multirow[t]{2}{*}{0.50} \\
\hline No & 147 & 153 & \\
\hline The total number of included vertebrae & 495 & 467 & N.A. \\
\hline \multicolumn{4}{|l|}{ Sites of vertebra } \\
\hline Cervical & 7 & 5 & 0.93 \\
\hline
\end{tabular}


Table 2 The characteristic comparison of patients in the training and validation group (Continued)

\begin{tabular}{llll}
\hline Characteristics & The training group $(\boldsymbol{n}=\mathbf{2 0 4})$ & The validation group $(\boldsymbol{n}=\mathbf{2 0 4})$ & 222 \\
\hline Thoracic & 241 & 210 \\
Lumbar & 218 & 30 \\
Sacral & 29 &
\end{tabular}

Abbreviations: $S D$ standard deviation, N.A. not applicable

272), no vertebrae collapse $(61.76 \%, 168 / 272)$, complete vertebrae posterior wall $(58.09 \%, 158 / 272)$, no vertebral endplate fracture $(82.35 \%, 224 / 272)$, a Bilsky scale of 0 (77.21\%, 210/272), osteolytic lesions (82.35\%, 224/272), and normal load-bearing lines of spine $(88.24 \%, 240 /$ 272). Vascular cement leakage occurred in $26.47 \%$ (72/ 272) of patients. Among them, only $1 / 72$ patients with vascular cement leakage was symptomatic with the patient reporting mild dyspnea.

\section{Comparisons between the training and validation group}

We randomly extracted $3 / 4$ of all included patients as the training $(n=204)$ and validation group $(n=204)$, respectively. Table 2 shows baseline characteristic comparison between the two groups, which demonstrated that the distribution of the eleven potential risk predictors was similar and comparable. Regarding the primary outcome, $27.94 \%$ (57/204) of patients had vascular cement leakage in the training group and $25.00 \%(51 / 204)$ in the validation group $(P=0.50)$.

\section{Creation of the PUFHS}

In the training group, the LASSO method found that three of the eleven risk predictors, including primary cancer types, number of treated vertebrae levels, and vertebrae collapse, were significant and included in the PUFHS (Table 3). The coefficients of the three included predictors were assigned based on the estimates obtained from the multiple logistic regression analysis. Thus, the PUFHS was created as follows: $\mathrm{P}(\mathrm{Y}=1)=$ $e^{-2.67+0.26 \times 1+0.65 \times 2-0.30 \times 3} /\left(1+e^{-2.67+0.26 \times 1+0.65 \times 2-0.30}\right.$ $\left.{ }^{\mathrm{x} 3}\right)$. ' 1 ' indicates primary cancer types, ' $x$ 2' indicates number of treated vertebrae levels, and ' $x$ 3' indicates vertebrae collapse. The scores of the three predictors were assigned based on the original data. For example, if a spine metastasis patient with rapid growth cancer (3 points) had more than $50 \%$ vertebrae collapse (3 points) and one treated vertebra level (1 point), the vascular cement leakage probability of the patient was $\mathrm{P}(\mathrm{Y}=1)=$ $e^{-2.67+0.26 * 3+0.65 * 1-0.30 * 3}$

$(1+$ $\left.e^{-2.67+0.26 * 3+0.65 * 1-0.30 * 3}\right)=10.53 \%$. We further developed a calculator which can calculate the probability of vascular cement leakage in order to facilitate the clinical utility of the score (Supplementary material).

\section{Validation of the PUFHS}

Discrimination and calibration were performed both in the training and validation group. The AUROC was 0.71 in the training group (Fig. 2) and 0.69 in the validation group (Table 4 and Fig. 3), which demonstrated the PUFHS was a useful and good model. The corresponding correct classification rates were 73.0 and $70.1 \%$, respectively. The discrimination slope was 0.12 (95\% CI: $0.078-0.17, P<0.001$ ) in the training group (Fig. 4) and 0.096 (95\% CI: $0.049-0.14, P<0.001$ ) in the validation group (Fig. 5), which indicated the mean risk differences between positive and negative events were significant in the two groups. The sensitivity and specificity was 24.6 and $91.8 \%$, respectively, in the training group and 29.4 and $83.7 \%$, respectively, in the validation group.

Considering the calibration ability, the slope was 0.78 (95\% CI: 0.45-1.10) in the training group (Fig. 6) and

Table 3 The PUFHS

\begin{tabular}{llc}
\hline Characteristics included in the PUFHS & Scores & Estimates $^{\mathbf{b}}$ \\
\hline Intercept & & -2.67 \\
Primary cancer types & 1 & 0.26 \\
$\quad$ Slow growth & 2 & \\
Moderate growth & 3 & \\
Rapid growth & & 0.65 \\
Number of treated vertebrae levels & 1 & \\
1 & 2 & \\
2 & 3 & -0.30 \\
3 & 4 & \\
$\geq 4$ & & \\
Vertebrae collapse & 1 & \\
No collapse & 2 & \\
Less than $50 \%$ & 3 & \\
More than $50 \%$ &
\end{tabular}

Notes: The PUFHS was created as follows:

$\mathrm{P}(\mathrm{Y}=1)=e^{-2.67+0.26 \times 1+0.65 \times 2-0.30 \times 3} /\left(1+e^{-2.67+0.26 \times 1+0.65 \times 2-0.30 \times 3}\right) ; x 1$ indicates primary cancer types; $x 2$ indicates number of treated vertebrae levels; $x 3$ indicates vertebrae collapse

An example was given as follows: if a spine metastasis patient with rapid growth cancer ( 3 points) had more than $50 \%$ vertebrae collapse ( 3 points) and one treated vertebra level (1 point), the vascular cement leakage probability of the patient was $\mathrm{P}(\mathrm{Y}=1)=e^{-2.67+0.26 * 3+0.65 * 1-0.30 * 3 /}$ $\left(1+e^{-2.67+0.26 * 3+0.65 * 1-0.30 * 3}\right)=10.53 \%$

Abbreviations: PUFHS Peking University First Hospital Score ${ }^{a}$ indicates characteristics were included according to the Least Absolute Shrinkage and Selection Operator; ${ }^{{ }}$indicates estimates were calculated from logistic regression analysis 


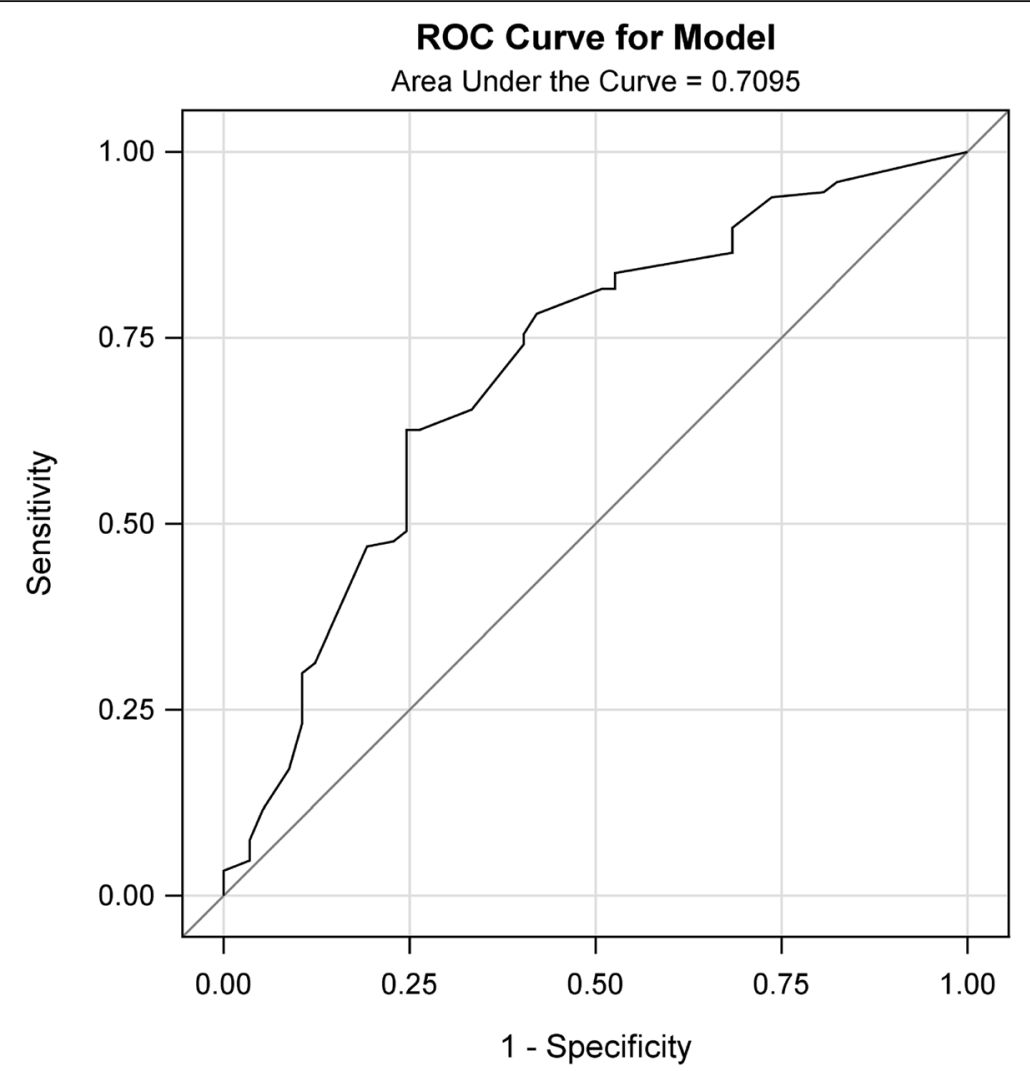

Fig. 2 The ROC curve for the PUFHS in the training group $(C$ value $=0.71)$

1.10 (95\% CI: $0.73-1.46)$ in the validation group (Table 5 and Fig. 7). The corresponding intercepts were 0.06 (95\% CI: - 0.04-0.17) and - 0.0079 (95\% CI: - 0.110.092), respectively, both of which were close to 0 . The $P$-values obtained from the goodness-of-fit test were both more than 0.05 in the two groups. These results, as mentioned above, indicated the PUFHS had good calibration ability. Table 6 shows the observed and predicted probability according to decile in the training and validation group. According to the predicted probabilities in each decile, patients were divided into three risk groups: the low, medium, and high group. The low risk group had a probability of less than $20 \%$ for vascular cement leakage, the medium risk group had a probability of $20 \%$ or more and less than $40 \%$, and the high risk group had a probability of $40 \%$ or more. The observed and predicted probabilities of vascular cement leakage were both significant different among the three risk groups $(P<0.01)$.

\section{Discussion}

The study found three risk predictors, namely, primary cancer types, number of treated vertebrae levels, and vertebrae collapse, were significantly associated with vascular cement leakage in spine metastases following percutaneous vertebroplasty. We further created the PUFHS with based on the above three risk predictors. The PUFHS was simple since it had only three variables. The predictive performance of the PUFHS was evaluated both in the training and validation group. The AUROC was 0.71 in the training group and 0.69 in the validation group, which demonstrated the PUFHS was a good and useful model. The calibration slope was near to $1(0.78$ in the training group and 1.10 in the validation group)

Table 4 The discrimination performances of the PUFHS in the training and validation group

\begin{tabular}{lllllll}
\hline Evaluation analysis & AUROC & CCR & Slope & $\mathbf{9 5 \%}$ Cl & Sensitivity & Specificity \\
\hline The training group & 0.71 & $73.0 \%$ & 0.12 & $0.078-0.17$ & $24.6 \%$ & $91.8 \%$ \\
The validation group & 0.69 & $70.1 \%$ & 0.096 & $0.049-0.14$ & $29.4 \%$ & $83.7 \%$ \\
\hline
\end{tabular}

Abbreviations: PUFHS Peking University First Hospital Score, $A U R O C$ area under the receiver operating characteristic curve, $C C R$ correct classification rate, $C I$ confidence interval 


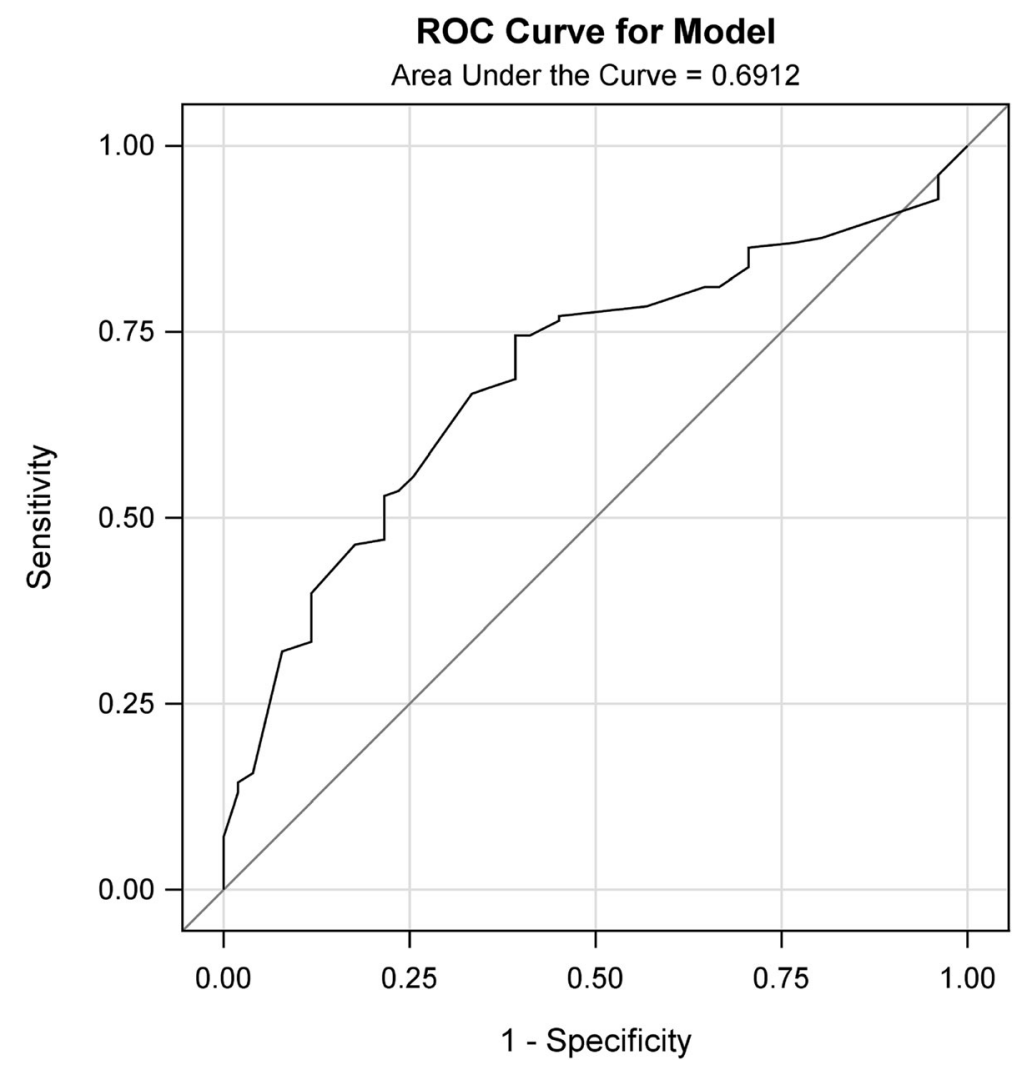

Fig. 3 The ROC curve for the PUFHS in the validation group ( $C$ value $=0.69$ )

and the intercepts (0.06 in the training group and0.0079 in the validation group) were close to 0 , which revealed the PUFHS also had good calibration ability. Therefore, the PUFHS can be a useful tool to realize early detection of vascular cement leakage and inform surgeons about the risk in advance. Furthermore, we developed a calculator which can calculate the probability of vascular cement leakage in order to facilitate the clinical utility of the score (see Supplementary material). Based on the predicted probabilities, patients were divided into three risk groups: the low, medium, and high groups. Among the three groups, the high risk group had the highest probability of vascular cement leakage (40\% or more), thus careful surgical preparation and intraoperative operation should be especially emphasized in those patients.

Of all the patients in the study, vascular cement leakage occurred in $26.47 \%$ of patients and this number was consistent with other studies. Corcos et al. [15] reported $25 \%$ of patients had vascular cement leakage after analyzing 56 cancer patients. Trumm et al. [16] found $25.5 \%$ of treated vertebrae occurred vascular leaks into segmental veins and $21.6 \%$ leaks into basivertebral veins after analyzing 202 malignant tumor patients. Pulmonary cement embolisms were observed after $7.8 \%$ of the procedures with follow-up of the X-ray of chest. Notably, vascular cement leakage was strongly associated with pulmonary embolism [17]. However, Barragán-Campos et al. [17] reported 423 cement leakages were identified in 117 patients and $78.5 \%$ of them were vascular. Inherent heterogeneity among spine metastatic lesions and technical diversity including PMMA-injecting volume, flow, and viscosity could lead to the difference.

Some studies also reported several risk factors were significantly associated with vascular cement leakage. Corcos et al. revealed [15] prior treatment and vertebral collapse were correlated with vascular cement leakage. Our study also found vertebral collapse was inversely correlated with vascular cement leakage, which was consistent with the study conducted by Corcos and his colleagues [15]. However, preoperative treatment was not found to be significant in our study. Corcos and his colleagues reckoned reduction in intravertebral pressure and vertebral vascularity after previous treatments could explain the role of prior treatment in preventing vascular cement leakage. We speculated heterogeneous definition of prior treatment might cause the difference. 


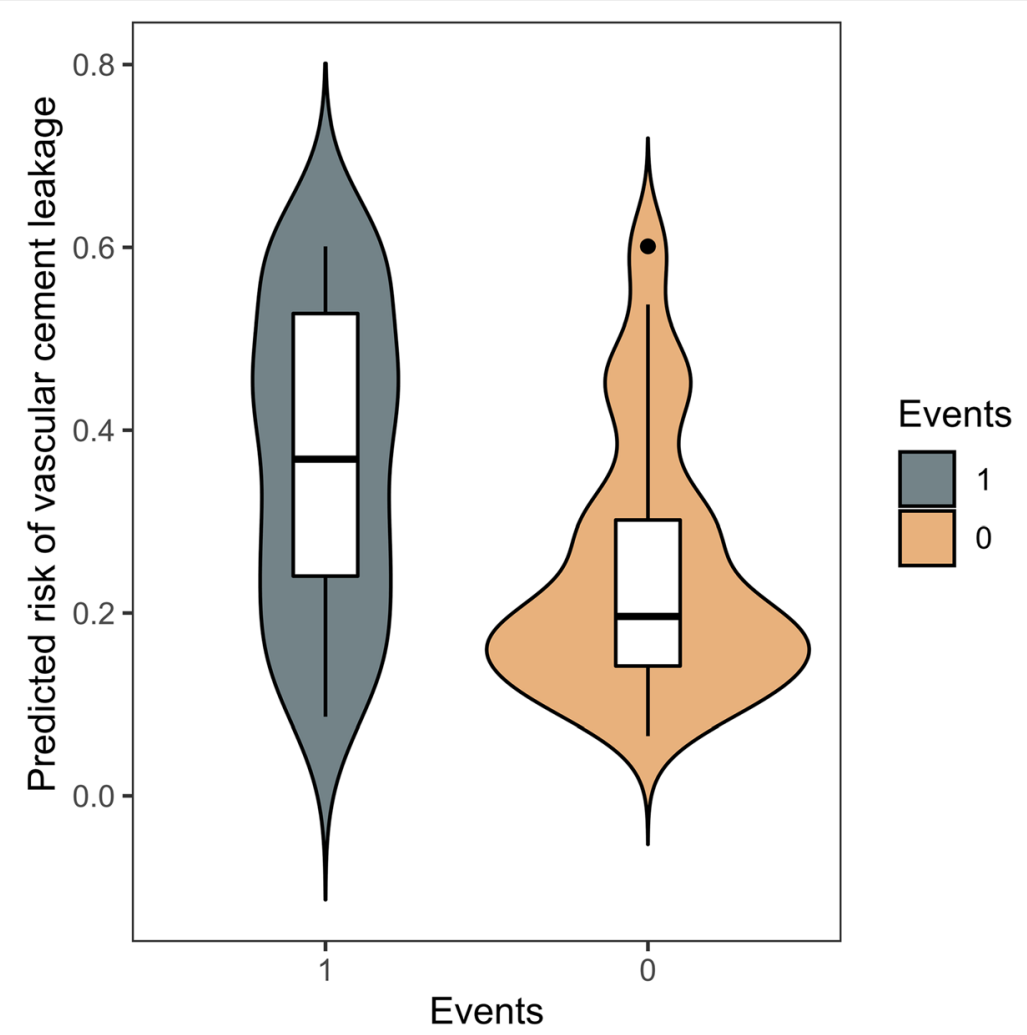

Fig. 4 The violin plot for the PUFHS in the training group (Discrimination slope $=0.12, P<0.001$ ). ' 1 ' indicates positive event (vascular cement leakage) and ' 0 ' indicates negative event (nonvascular cement leakage)

In our study, primary cancer type was included in the PUFHS. Rapid growth cancers were more likely to suffer from vascular cement leakage. Reidy et al. [18] found vertebrae containing simulated metastatic tumor could significantly increase intravertebral body pressures during percutaneous vertebroplasty as compared with intact vertebrae. Higher intravertebral body pressures could lead to more cement leakages. Besides, vertebral vascularity could definitely affect vascular cement leakage since hypervascularity provided more ways for PMMA to leak. Thus, high intravertebral pressures, resulted from rapid growth of metastatic cancers, and abundant vertebral vascularity could justify the results. The number of treated vertebrae segments was also proved to be significant simply because the more surgically treated segments indicated the greater the possibility of cement leakage.

Previously, we proposed an algorithm based on the treated vertebrae level, cortical osteolytic destruction in the posterior wall, and the Bilsky scale, which can calculate cement injection volumes in spine metastases treated with percutaneous vertebroplasty [19]. This algorithm can help surgeons to guide surgical planning and cement injections. However, the algorithm still cannot early predict cement leakage and thus prevention strategies cannot be performed in advance. In the present study, we created the PUFHS, making early detection of vascular cement leakage a reality. Besides, classifying patients in the low, medium, and high risk group contributes to enhanced quality of healthcare. We aimed at developing an algorithm especially to calculate the probability of vascular cement leakage because the multitude of patients only had one vertebra metastasis and sometimes we found vascular cement leakage in the vein but we cannot distinguish which vertebra the cement leakage came from particularly in cases treating with multiple vertebra percutaneous vertebroplasty. Thus we performed the analysis per patient rather than per vertebra, this might add precision.

This study had several limitations. First, the study was retrospective and enrolled patients in a single medical center, so selection bias would definitely exist. Second, some risk variables, such as PMMA-injecting volume, flow, and viscosity, which could influence vascular cement leakage [20], were not assessed in the study. Therefore, although the PUFHS showed good discrimination and calibration ability, the PUFHS still need large prospective sample to be validated. 


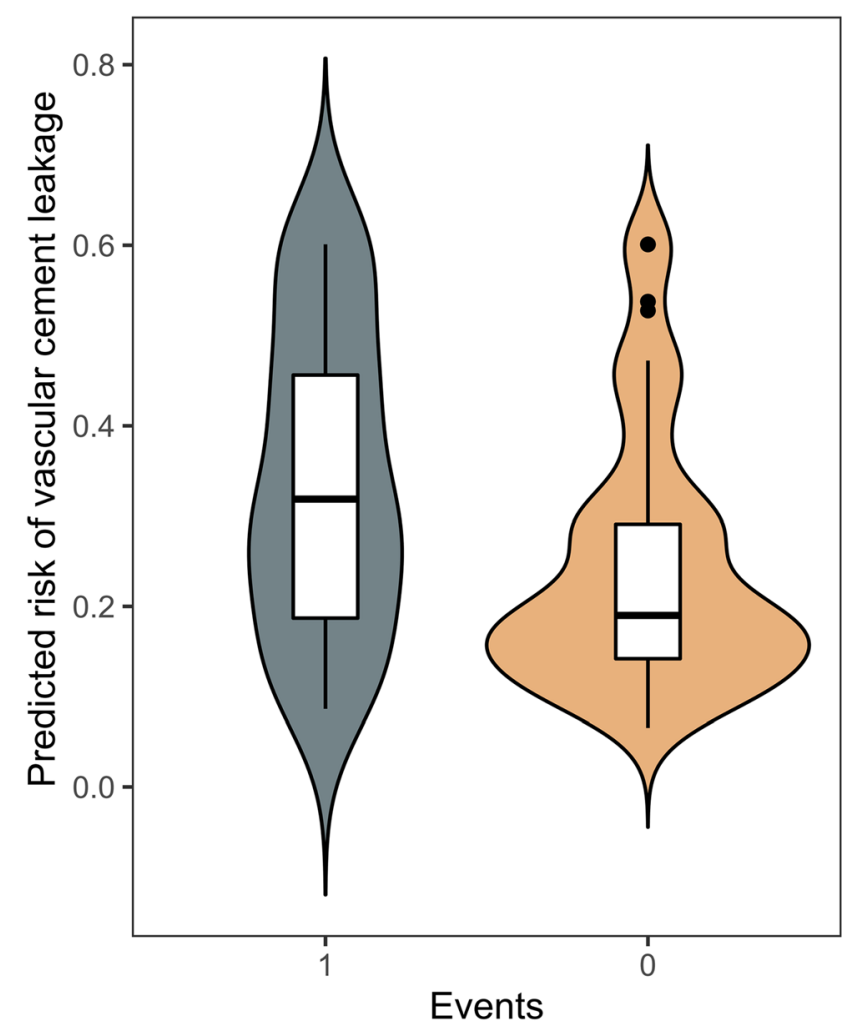

\section{Events}

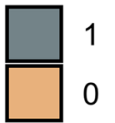

leakage) and ' 0 ' indicates negative event (nonvascular cement leakage)

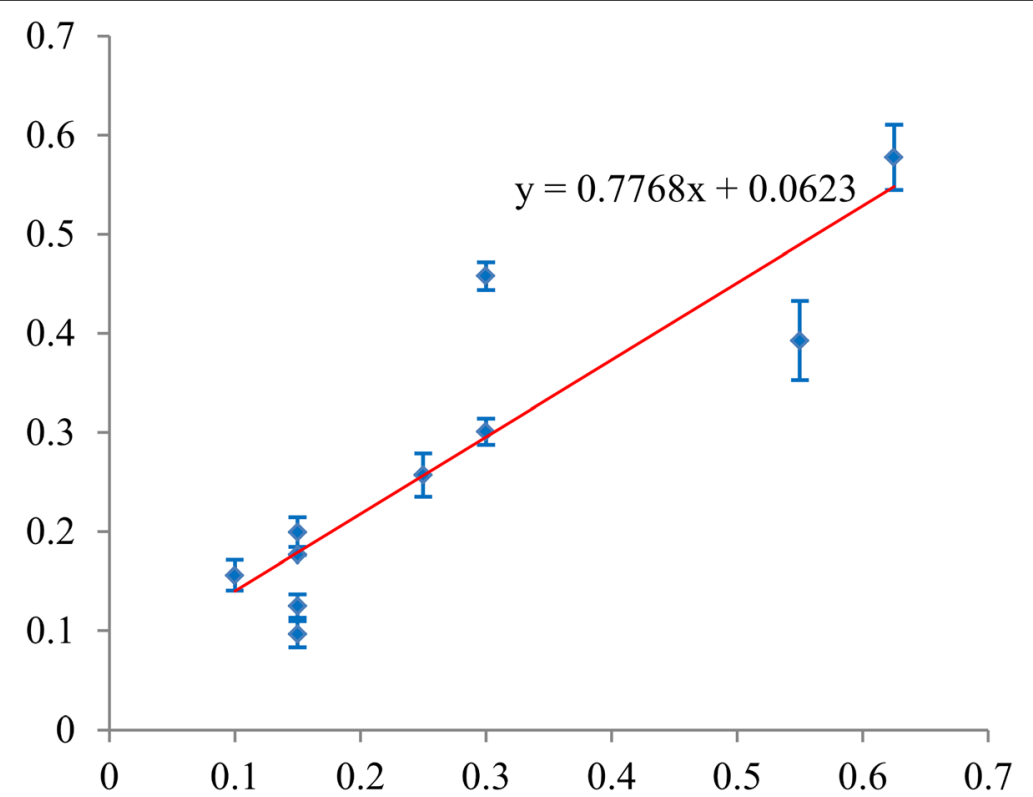

Fig. 6 Plotting decile of the PUFHS-predicted probability of vascular canal cement leakage against the observed proportions in the training group (Calibration slope $=0.78$ and intercept $=0.06$ ). The red line indicates the smooth was fitted using linear regression analysis in the Microsoft Excel software 
Table 5 The calibration performances of the PUFHS in the training and validation group

\begin{tabular}{llllll}
\hline Evaluation analysis & Slope & $\mathbf{9 5 \%} \mathbf{C l}$ & Intercept & $\mathbf{9 5 \%} \mathbf{C l}$ & Goodness-of-Fit test \\
\hline The training group & 0.78 & $0.45-1.10$ & 0.06 & $-0.04-0.17$ & 0.98 \\
The validation group & 1.10 & $0.73-1.46$ & -0.0079 & $-0.11-0.092$ & 0.16
\end{tabular}

Abbreviations: PUFHS Peking University First Hospital Score, Cl confidence interval

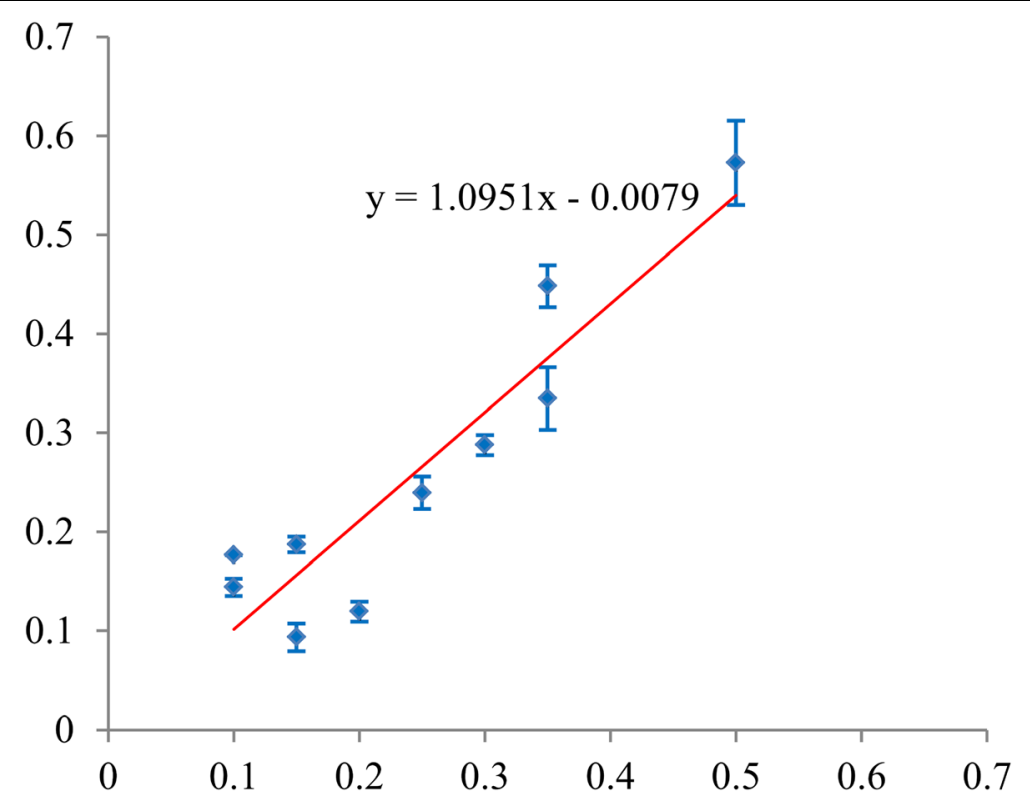

Fig. 7 Plotting decile of the PUFHS-predicted probability of vascular canal cement leakage against the observed proportions in the validation group (Calibration slope $=1.10$ and intercept $=-0.0079$ ). The red line indicates the smooth was fitted using linear regression analysis in the Microsoft Excel software

Table 6 Observed and predicted probability according to decile in the training and validation group

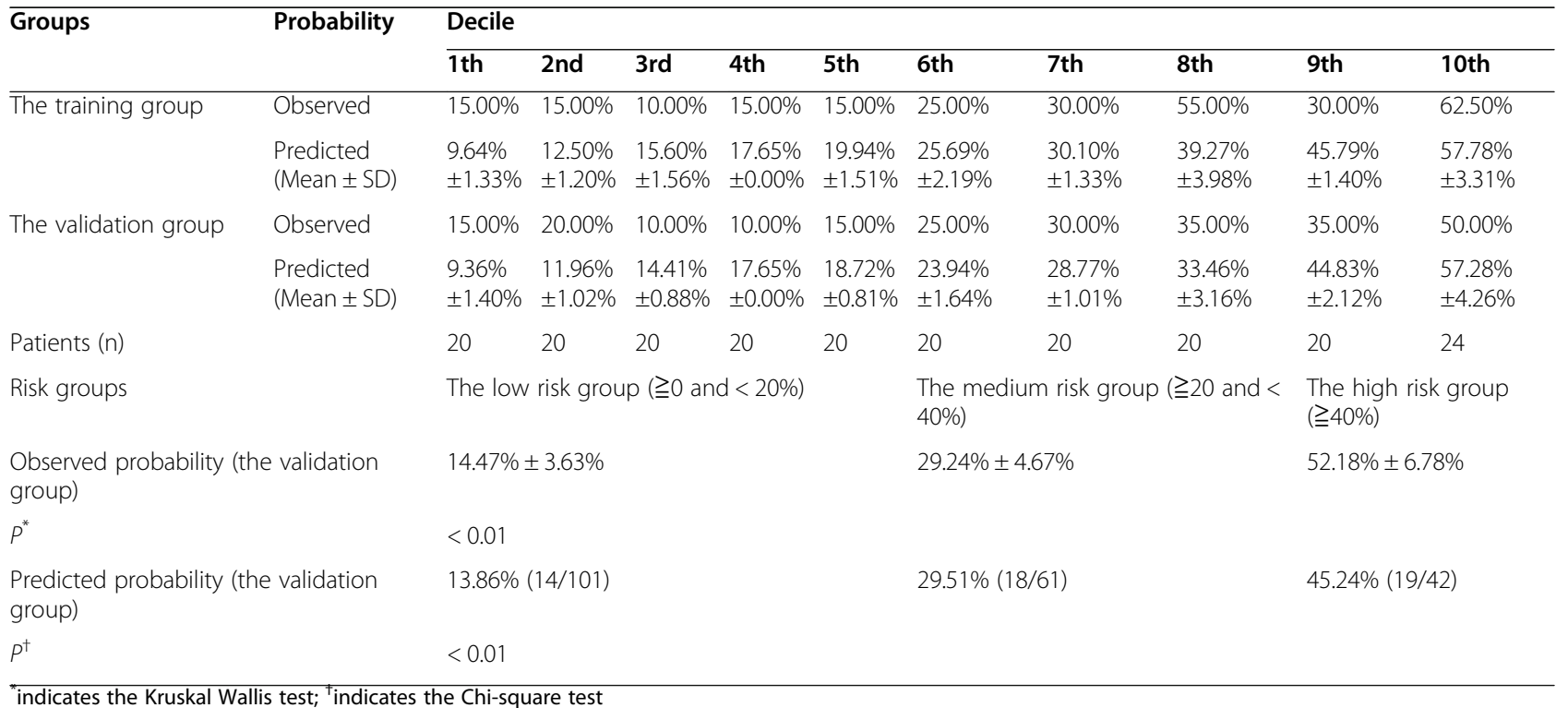




\section{Conclusion}

Vascular cement leakage is common among spine metastases after percutaneous vertebroplasty. The PUFHS can calculate the probability of vascular cement leakage, which can be a useful tool to inform surgeons about vascular cement leakage risk in advance.

\section{Abbreviations}

AUROC: Area under the receiver operating characteristic curve; CCR: Correct classification rate; $\mathrm{Cl}$ : Confidence interval; PUFHS: Peking University First Hospital Score; LASSO: Least Absolute Shrinkage and Selection Operator; N.A: Not applicable; SD: Standard deviation

\section{Supplementary Information}

The online version contains supplementary material available at https://doi. org/10.1186/s12885-021-08503-2.

\section{Additional file 1.}

\section{Acknowledgements}

None.

\section{Authors' contributions}

SDX and YPC designed the study. YXP, BW, and MXL wrote and revised the manuscript. SDX, YPC, YXP, BW, and MXL revised the manuscript. All authors read and approved the final manuscript.

\section{Funding}

Not applicable.

\section{Availability of data and materials}

The datasets of the current study are available under reasonable request.

\section{Declarations}

\section{Ethics approval and consent to participate}

All analyses of human data conducted in this study were approved by the Ethics Committee Board of the Peking University First Hospital and in accordance with the ethical standards of the institutional and/or national research committee and with the 1964 Helsinki declaration and its later amendments or comparable ethical standards. Informed consent was waived by the Ethics Committee Board of the Peking University First Hospital that approved the study because of the retrospective nature of the study.

\section{Consent for publication}

Not applicable.

\section{Competing interests}

The authors declare that they have no competing interests.

\begin{abstract}
Author details
${ }^{1}$ Department of Orthopedic Surgery, Peking University First Hospital, No.8 Xishiku Street, Xicheng District, Beijing 100032, China. ${ }^{2}$ Department of Orthopedic Surgery, Hainan Hospital of Chinese PLA General Hospital, Haitang District, Jianglin Rd, Sanya 572013, China. ${ }^{3}$ Graduate School of Chinese PLA Medical College, No. 28 Fuxing Road, Haidian District, Beijing 100853, China.
\end{abstract}

Received: 22 April 2021 Accepted: 15 June 2021

Published online: 02 July 2021

\section{References}

1. Bilsky MH. New therapeutics in spine metastases. Expert Rev Neurother. 2005;5(6):831-40. https://doi.org/10.1586/14737175.5.6.831.

2. Qian Z, Sun Z, Yang H, Gu Y, Chen K, Wu G. Kyphoplasty for the treatment of malignant vertebral compression fractures caused by metastases. J Clin Neurosci. 2011;18(6):763-7. https://doi.org/10.1016/j.jocn.2010.09.021.
3. Shimony JS, Gilula LA, Zeller AJ, Brown DB. Percutaneous vertebroplasty for malignant compression fractures with epidural involvement. Radiology. 2004;232(3):846-53. https://doi.org/10.1148/radiol.2323030353.

4. Kushchayev SV, Wiener PC, Teytelboym OM, Arrington JA, Khan M, Preul MC. Percutaneous Vertebroplasty: a history of procedure, technology, culture, specialty, and economics. Neuroimaging Clin N Am. 2019;29(4):48194. https://doi.org/10.1016/j.nic.2019.07.011.

5. Lee SK, Weiss B, Yanamadala V, Brook A. Percutaneous interventional Management of Spinal Metastasis. Semin Interv Radiol. 2019;36(3):249-54. https://doi.org/10.1055/s-0039-1694698.

6. Delgado-Lopez PD, Roldan-Delgado H, Corrales-Garcia EM. Stereotactic body radiation therapy and minimally invasive surgery in the management of spinal metastases: a change in the paradigm. Neurocirugia (Astur). 2020; 31(3):119-31.

7. Sorensen ST, Kirkegaard AO, Carreon L, Rousing R, Andersen MO. Vertebroplasty or kyphoplasty as palliative treatment for cancer-related vertebral compression fractures: a systematic review. Spine J. 2019;19(6): 1067-75. https://doi.org/10.1016/j.spinee.2019.02.012.

8. Kim YJ, Lee JW, Park KW, Yeom JS, Jeong HS, Park JM, et al. Pulmonary cement embolism after percutaneous vertebroplasty in osteoporotic vertebral compression fractures: incidence, characteristics, and risk factors. Radiology. 2009;251(1):250-9. https://doi.org/10.1148/radiol.2511080854.

9. Chew C, Craig L, Edwards R, Moss J, O'Dwyer PJ. Safety and efficacy of percutaneous vertebroplasty in malignancy: a systematic review. Clin Radiol. 2011;66(1):63-72. https://doi.org/10.1016/j.crad.2010.09.011.

10. Fadili Hassani S, Cormier E, Shotar E, Drir M, Spano JP, Morardet L, et al. Intracardiac cement embolism during percutaneous vertebroplasty: incidence, risk factors and clinical management. Eur Radiol. 2019;29(2):66373. https://doi.org/10.1007/s00330-018-5647-0.

11. D'Errico S, Niballi S, Bonuccelli D. Fatal cardiac perforation and pulmonary embolism of leaked cement after percutaneous vertebroplasty. J Forensic Legal Med. 2019;63:48-51. https://doi.org/10.1016/j.jflm.2019.03.004.

12. Tokuhashi $Y$, Matsuzaki H, Oda H, Oshima M, Ryu J. A revised scoring system for preoperative evaluation of metastatic spine tumor prognosis. Spine (Phila Pa 1976). 2005;30(19):2186-91. https:/doi.org/10.1097/01.brs.0000180401.06919.a5.

13. Gabriel C, Jonathan D, Charles M, Sandrine L, Anne A, Thierry DB, et al. Cement leakage in percutaneous vertebroplasty for spinal metastases: a retrospective evaluation of incidence and risk factors. Spine. 2014;39(5):332-8.

14. Bilsky MH, llya L, Fourney DR, Michael G, Schmidt MH, Peter Paul V, et al. Reliability analysis of the epidural spinal cord compression scale. J Neurosurg Spine. 2010;13(3): 324-8. https:/doi.org/10.3171/2010.3.SPINE09459.

15. Corcos G, Dbjay J, Mastier C, Leon S, Auperin A, De Baere T, et al. Cement leakage in percutaneous vertebroplasty for spinal metastases: a retrospective evaluation of incidence and risk factors. Spine (Phila Pa 1976). 2014;39(5):E332-8. https://doi.org/10.1097/BRS.0000000000000134.

16. Trumm CG, Pahl A, Helmberger TK, Jakobs TF, Zech CJ, Stahl R, et al. CT fluoroscopy-guided percutaneous vertebroplasty in spinal malignancy: technical results, PMMA leakages, and complications in 202 patients. Skelet Radiol. 2012;41(11):1391-400. https://doi.org/10.1007/s00256-012-1365-X.

17. Barragan-Campos HM, Vallee JN, Lo D, Cormier E, Jean B, Rose M, et al. Percutaneous vertebroplasty for spinal metastases: complications. Radiology. 2006;238(1):354-62. https://doi.org/10.1148/radiol.2381040841.

18. Reidy D, Ahn H, Mousavi P, Finkelstein J, Whyne CM. A biomechanical analysis of intravertebral pressures during vertebroplasty of cadaveric spines with and without simulated metastases. Spine (Phila Pa 1976). 2003;28(14): 1534-9. https://doi.org/10.1097/01.BRS.0000076828.33308.5F.

19. Cui $Y$, Pan $Y$, Lei M, Mi C, Wang B, Shi $X$. The first algorithm calculating cement injection volumes in patients with spine metastases treated with percutaneous Vertebroplasty. Ther Clin Risk Manag. 2020;16:417-28. https:// doi.org/10.2147/TCRM.S253046.

20. Anselmetti GC, Zoarski G, Manca A, Masala S, Eminefendic H, Russo F, et al. Percutaneous vertebroplasty and bone cement leakage: clinical experience with a new high-viscosity bone cement and delivery system for vertebral augmentation in benign and malignant compression fractures. Cardiovasc Intervent Radiol. 2008:31(5):937-47. https://doi.org/10.1007/s00270-0089324-6.

\section{Publisher's Note}

Springer Nature remains neutral with regard to jurisdictional claims in published maps and institutional affiliations. 